\title{
Super Star Clusters in M82
}

\author{
Robert W. O'Connell \\ Astronomy Department, University of Virginia, Charlottesville, VA \\ 22903, USA
}

\begin{abstract}
M82 is the nearest starburst galaxy. It contains two large systems of super star clusters, one being spawned today in the active starforming core, and one produced by an earlier starburst event which coincided with the last orbital passage of its neighbor, M81. The proximity of M82 makes it uniquely valuable for a wide range of studies of massive young clusters and their environments.
\end{abstract}

\section{Introduction: M82, The Nearest Starburst Galaxy}

M82 is the most remarkable nearby galaxy. Seen in the optical continuum bands, it would strike you as a mild-mannered, if slightly eccentric, edge-on disk system. Its main peculiarities are a conspicuous network of dust lanes; an unusually smooth texture at large radius for a disk galaxy; and strange, high surface brightness knots near its center. However, a more careful look with narrowband emission line filters, first obtained by Elvius (1962) and Lynds \& Sandage (1963), reveals a raging psychopath, with glowing plumes of hot gas extending along the minor axis to distances of $5 \mathrm{kpc}$.

The plumes stimulated intense scrutiny of M82 at all wavelengths from the radio to the $\mathrm{X}$-ray. The picture which has emerged is the following. During the last several $100 \mathrm{Myr}$ years, tidal interactions with its large spiral companion, M81, induced a concentrated starburst in M82 with a star formation rate of $\sim 10 \mathrm{M}_{\odot} \mathrm{yr}^{-1}$. Energy and gas injection from supernovae, at a rate of $\sim 0.1$ supernova $\mathrm{yr}^{-1}$, drive a large-scale galactic wind along the minor axis of M82. All of the bright radio and infrared sources associated with the active starburst (age $\lesssim 20 \mathrm{Myr}$ ) are confined within a radius of $\sim 250 \mathrm{pc}$ of the galaxy's center. Most of this volume is heavily obscured by dust at optical wavelengths but is easily probed in the infrared and radio.

Although much of the effort to date on M82 has been directed at the starburst itself and its complex interstellar medium, M82 is also a key fiducial for the study of super star clusters. Not only is it the nearest galaxy with a large system of young super star clusters $(\mathrm{D}=3.6 \mathrm{Mpc}$, linear scale $17.5 \mathrm{pc} / \mathrm{arcsec})$, but also it contains two such systems: one being created in the ongoing starburst and an older (600 Myr) one now well into its mid-life evolution. 


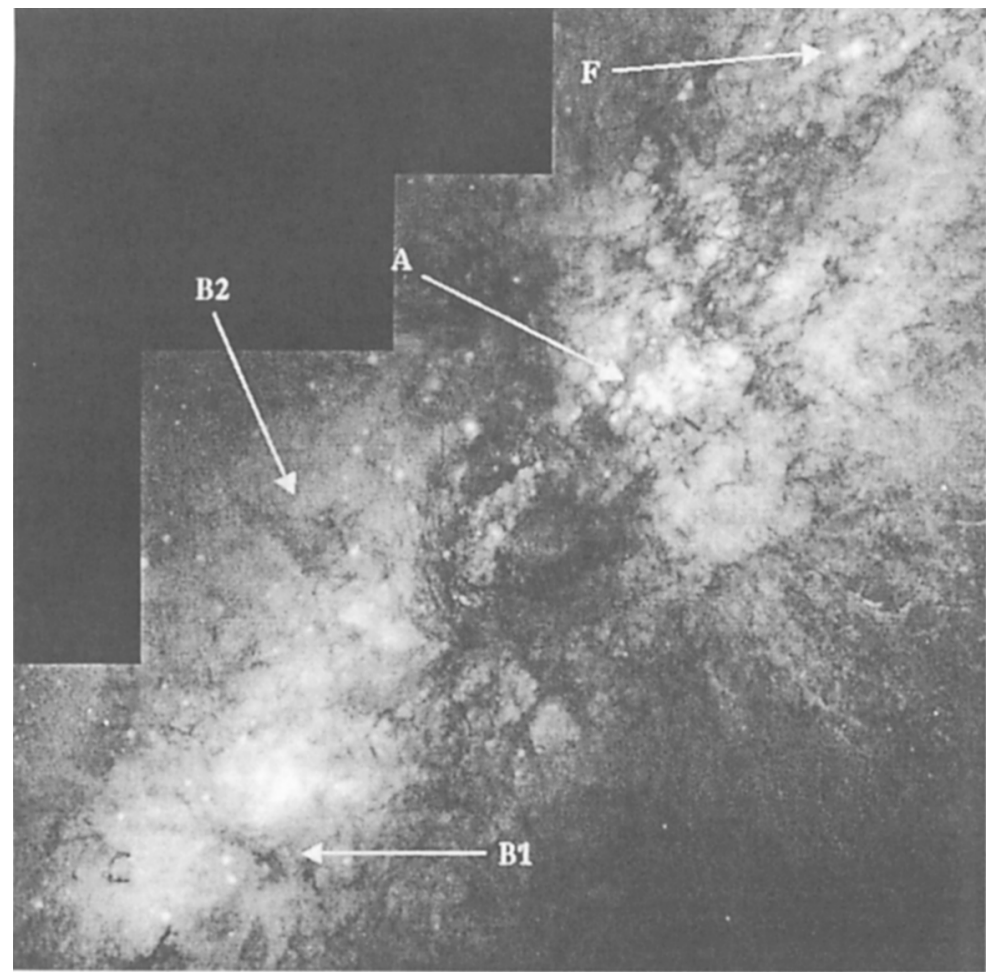

Figure 1. The central regions of M82 at $0.1^{\prime \prime}$ resolution. Composite image from HST/WFPC-2 B, V, and I band frames. About $120^{\prime \prime}$ of the major axis is shown, running diagonally across the frame (from NE in lower left to SW in upper right). Areas discussed in the text are identified. A full-color version of this image is available from STScI (PR-01-08).

\section{Young Super Star Clusters in the Active Starburst}

The central regions of M82 are astonishingly complex (see Figure 1). The first studies of the brighter knotty structures by van den Bergh (1971) and O'Connell \& Mangano (1978, hereafter OM78) showed that these were luminous, young, compact star clusters or cluster complexes. The brightest complex, region A, exhibited substructure to the seeing limit and, after a conservative extinction correction, had a remarkably bright mean surface brightness of $\mu_{\mathrm{V}} \sim 14.5$ $\mathrm{mag} / \operatorname{arcsec}^{2}$, comparable to those of normal galactic nuclei. Spectral synthesis indicated it contains stars as young as $5 \mathrm{Myr}$. Its integrated intrinsic $M_{\mathrm{V}}$ is $\lesssim-17.5$. The M82 knots were perhaps the first recognized super star clusters, and in fact van den Bergh (1971) used the term "superclusters" to describe them.

HST-Planetary Camera imaging by O'Connell et al. (1995) resolved complex A and its surroundings into a swarm of over 100 individual bright clusters. 
These have typical FWHM of $0.2^{\prime \prime}$ or 3.5 pc. The mean intrinsic brightness of the cluster sample is $M_{\mathrm{V}} \sim-11.6\left(4 \times 10^{6} \mathrm{~L}_{\odot, \mathrm{V}}\right)$; the brightest cluster has $M_{\mathrm{V}} \sim-13.2$ (much brighter than the most luminous cluster in the Local Group, 30 Dor/R136, at -11.1).

Infrared and radio observations, reviewed by Telesco (1988) and Kronberg (1988), show that most of the starburst core in M82 lies behind thick dust lanes and is not detectable in the visible bands. Satyapal et al. (1997) identified 12 compact sources with ground-based K-band imaging within the core and obtained ages in the range 6-10 Myr for these. Undoubtedly, HST-resolution IR imaging would resolve these into many individual clusters, as in the case of region A. Recent IR spectroscopy by Förster Schreiber et al. (2001) suggests remarkably dense packing of the ionizing sources, presumably the clusters containing OB stars, with typical separation $\lesssim 10$ pc. O'Connell et al. (1995) argued that region $\mathrm{A}$ is, in fact, part of the starburst core which has relatively low extinction along the line of sight. Since dust is mixed throughout the core, we can see a fractional volume $\sim 1 / \tau(\lambda)$ at any wavelength, which for the range of estimated extinctions is $\sim 0.03-0.20$ at V. Over 2000 M82A-like super star clusters could be present within the starburst core. There appears to be a direct link between the hot gas within region $\mathrm{A}$ and the base of the $\mathrm{H} \alpha$-bright galactic wind, implying that the visible clusters help drive the wind.

Controversy about the IMF within the core has persisted. The small $\mathrm{M} / \mathrm{L}_{\text {bol }}$ ratio estimated from IR observations suggests that the IMF is truncated or otherwise deficient in low mass stars (e.g. Rieke et al. 1993). However, alternative determinations of extinction and allowance for the inhomogeneity of the core produce ratios in agreement with normal IMF's (e.g. Satyapal et al. 1997). OM78 found from the emission line rotation curve that the clusters within $\mathrm{A}$ have $\mathrm{M} / \mathrm{L}_{\mathrm{V}} \sim 0.1$ within a factor of 2 , consistent with young systems with normal IMF's.

The most remarkable individual cluster in M82 is object F (OM78), lying about $500 \mathrm{pc}$ from region A near the western edge of the starburst core. With a corrected $M_{\mathrm{V}} \sim-14.5$, this was the most luminous star cluster known until the record was broken in NGC 7252 (Schweizer \& Seitzer 1993). Gallagher \& Smith (1999) and Smith \& Gallagher (2001) obtained high resolution spectroscopy of F. From synthesis models, they were able to assign an age of $60 \pm 20 \mathrm{Myr}$ (intermediate between the starburst and the clusters in M82 B described below) and derived from the velocity dispersion $(13.4 \mathrm{~km} / \mathrm{s})$ a mass of $1.2 \times 10^{6} \mathrm{M}_{\odot}$. The resulting $\mathrm{M} / \mathrm{L}_{\mathrm{V}}$ ratio is too small by a factor of 5 to be consistent with the derived age. After considering all the uncertainties, Smith \& Gallagher conclude that the IMF in this cluster must be top-heavy with a lower mass limit of 2-3 $\mathrm{M}_{\odot}$. Because of mass loss during stellar evolution, the cluster is unlikely to survive more than $\sim 2$ Gyr.

\section{Intermediate Age Clusters in the Fossil Starburst}

There is now good evidence that M82 suffered at least two starburst episodes. Region B, extending 400-1000 pc NE of the galaxy's center, has an abnormally high surface brightness and the A/F-type absorption line spectrum associated with post-starburst systems. OM78 proposed that this was the site of an ear- 
lier starburst in M82. Using HST/WFPC2 images, we (de Grijs, O'Connell, \& Gallagher 2001) identified over 100 luminous, resolved cluster candidates in region B. From B,V,I photometry we were able to simultaneously solve for each cluster's age and extinction. The clusters have intrinsic luminosities in the range $10^{4-6} \mathrm{~L}_{\odot, \mathrm{V}}$, significantly fainter than the objects in region $\mathrm{A}$ (as expected if they are older). The brightest cluster (visible in Fig. 1 near the dust lane at the edge of region B2) has a corrected $M_{\mathrm{V}}=-10.6$. The age distribution in both regions $\mathrm{B} 1$ and B2 is broad, but in each there is a sharp peak at age $\sim 600 \mathrm{Myr}$ (see the de Grijs et al. poster at this meeting). Independent dynamical modeling of the HI debris in the M81-M82-NGC3077 system by Brouillet et al. (1991) predicts that the last near encounter between M81 and M82 occurred $~ 500$ Myr ago, coincident with the M82 B age peak. This suggests that cluster formation in B was induced by the tidal passage. Since that time, cluster formation has been largely suppressed in region $\mathrm{B}$.

M82 is the only large system of super star clusters where the individual members are significantly resolved by HST. In region B we obtain core radii in the range 2-8 pc. The luminosity function, corrected to a constant age of 50 Myr is similar to that in M82A and other young super star cluster systems and consistent with expectations for the progenitors of massive globular clusters. It has a power law slope of $\alpha=-1.2$, somewhat flatter than those of most other systems, perhaps suggesting that dynamical destruction can be detected.

\section{Conclusion}

M82 is unique not for the magnitude of its activity but rather for its proximity. Starbursts of this scale are likely to be common features of early galaxy evolution, and M82 is the nearest analogue to the intriguing sample of star-forming galaxies recently identified (e.g. by their Lyman dropouts) at redshifts $z 3$ (e.g. Steidel et al. 1996; Lowenthal et al. 1997). M82 provides a close-up view of both an active starburst and also, in region $\mathrm{B}$, of the subsequent post-burst phase which is apparently responsible for the " $\mathrm{E}+\mathrm{A}$ " or "quenched" spectra which are encountered in Butcher-Oemler effect galaxy clusters at high redshift (e.g. Oemler 1992; Couch et al. 1998). There are large numbers of super star clusters associated with each phase. Other nearby galaxies are known to exhibit one or another of these features, but none affords the opportunity to study both at such close range, or with such a wealth of correlative data, as does M82.

Among the astrophysical issues which can be explored in M82 are the following: cluster structures (morphologies, gradients); the stellar content of clusters (including CMD's in the exteriors); the IMF; cluster dynamical evolution; propagating star formation based on age-dating of clusters; metallicity-age-position correlations; the structure of the ISM at high spectral resolution using clusters as background sources; interactions between clusters in dense environments; and cluster winds and the transition between these and the galactic wind. 


\section{References}

Brouillet, N., Baudry, A., Combes, F., Kaufman, M., \& Bash, F. 1991, A\&A, 242,35

Couch, W.J., Barger, A.J., Smail, I., Ellis, R.S., \& Sharples, R.M. 1998, ApJ, 497, 188

de Grijs, R., O'Connell, R.W., \& Gallagher, J.S. 2001, AJ, 121, 768 (\& poster at this conference)

Elvius, A. 1962, Lowell Obs Bull No. 119

Förster Schreiber, N.M., Genzel, R., Lutz, D., Kunze, D., \& Sternberg, A. 2001, ApJ, 552, 544

Gallagher, J.S., \& Smith, L.J. 1999, MNRAS, 304, 540

Kronberg, P.P. 1988, in Galactic and Extragalactic Star Formation, ed. R.A. Pudritz \& M. Fich (Dordrecht: Kluwer), 391

Lowenthal, J.D., Koo, D.C., Guzman, R., et al. 1997, ApJ, 481, 673

Lynds, C.R., \& Sandage, A.R. 1963, ApJ, 137, 1005

O'Connell, R.W., Gallagher, J.S., Hunter, D.A., \& Colley, W.N. 1995, ApJ, 446, L1

O'Connell, R.W., \& Mangano, J.J. 1978, ApJ, 221, 62

Oemler, A. 1992, in Clusters \& Superclusters of Galaxies, ed. A.C. Fabian (Dordrecht: Kluwer), p. 29

Rieke, G.H., Loken, K., Rieke, M.J., \& Tamblyn, P. 1993, ApJ, 412, 99

Satyapal, S., Watson, D.M., Pipher, J.L., et al. 1997, ApJ, 483, 148

Smith, L.J, \& Gallagher, J.S. 2001, MNRAS, in press, (astro-ph/0104429; \& poster at this conference)

Steidel, C.C., Giavalisco, M., Pettini, M., Dickinson, M., \& Adelberger, K.L. 1996, ApJ, 462, L17

Schweizer, F., \& Seitzer, P. 1993, ApJ, 417, L29

Telesco, C.M., 1988, ARA\&A, 26, 343

van den Bergh, S. 1971, A\&A, 12, 474

\section{Discussion}

J. Frogel: Clarify the relative locations of (a) the dynamical nucleus; (b) the region with all of the $600 \mathrm{Myr}$ old clusters; and (c) the origin of the $\mathrm{H} \alpha$ gas that is being expelled from the galaxy.

R. O'Connell: The dynamical center is hard to pin down because of the complexity of the galaxy but is thought to coincide with the $2.2 \mu$ peak, which is about $2^{\prime \prime} \mathrm{NW}$ of region $\mathrm{A}$. The intermediate age clusters lie in the major axis area $400-1000 \mathrm{pc} \mathrm{NE}$ of region A. The minor axis plume is thought to consist of gas lost from individual massive stars and supernova remnants and a large amount of entrained matter from the ISM. Interestingly, a significant fraction of the $\mathrm{H} \alpha$ radiation from the plume is light scattered by dust grains, apparently photons from the starburst core. This complicates the kinematical interpreta- 
tion of the plume, and it's fair to say that a satisfactory model has not yet emerged.

B. Whitmore: What is the size of the $600 \mathrm{Myr}$ year old region? I would have expected differential rotation to have spread it out after $600 \mathrm{Myr}$, which is probably several orbital time scales. Is there evidence for clusters with similar age on the other side of the galaxy?

R. O'Connell: You're right that this is an interesting problem. The area we surveyed carefully for clusters was about $500 \mathrm{pc}$ in extent, but we examined the available HST and ground-based imagery of the rest of the galaxy and find that bright clusters like those in B are not common outside this region (and the core). We don't know how region B has managed to stay together, though this might be consistent with a solid body rotation curve.

A. Walker: Why did the central region of M82 wait $585 \mathrm{Myr}$ after the M81/M82 interaction before lighting up?

R.O'Connell: Another good question, for which we like to wave our hands and say that starbursts are probably self-quenched after 50-100 Myr years by supernova-driven outflows, which remove any remaining cool gas. Of course, we see one of these now in the active core. In the case of M82, there is a large amount of circumgalactic cold material orbiting from the tidal encounter, and a recent infall from this reservoir could have triggered the active event.

A. Ramirez Could the M82 F cluster be the nucleus of a galaxy which merged with M82?

R. O'Connell: We don't think there was an actual merger. The tidal encounter with M81 was not close. Of course, this doesn't rule out the possibility that M82 could have absorbed a low-mass member of the M81 group and even could have done so relatively recently. Cluster F may be at the extreme, but it still seems part of the continuum of super star cluster properties, rather than something distinct.

F. Schweizer: The M81/M82 system is complicated, since there is also a third galaxy (NGC 3077) participating in the interaction. Whose model is it that predicts an interaction with M81 about 600 Myr ago? And did this model specifically exclude a mass transfer, since such transfer are quite frequent in strong interactions?

R. O'Connell: The model I discussed was by Brouillet et al. (1991). It did indeed incorporate the full interaction of the three galaxies and reproduce the HI distribution within the M81-M82-N3077 system (though based on earlier data than the map I showed, which was from Yun, Ho, \& Lo 1994, Nature, 372, 530). The model predicted a closest passage by M81 at a distance of about 21 $\mathrm{kpc}$, which wouldn't necessarily induce a major gas transfer. OM78 argued that a transfer was unlikely because the total cold material near M82 was a large 
fraction (40\%) of the complement in M81 but was about the amount expected to be tidally disrupted from M82 itself if it had been a normal late-type disk galaxy before the encounter. Yun, Ho, \& Lo (1993, ApJ, 411, L17) found that the details of the HI map near M82 likewise suggest tidal disruption rather than transfer.

H. Lamers: You found a peak in the cluster formation rate at about $600 \mathrm{Myr}$ and little evidence for cluster formation in the intermediate age between about $15 \mathrm{Myr}$ and $500 \mathrm{Myr}$. But the cluster disruption time in such a violent system must be small. So clusters formed at intermediate ages may have been disrupted, and the peak at $600 \mathrm{Myr}$ was actually the last time when massive clusters were formed that survived disruption (apart from the young burst at $15 \mathrm{Myr}$ ).

R. O'Connell: Yes, we expected the inner regions of M82 to be a difficult environment for clusters to survive, but we were surprised to find a significant fraction $(22 \%)$ of the clusters in region B to be older than 1 Gyr. By contrast, we find only 4 younger than $100 \mathrm{Myr}$. Although the bright background is a serious problem, we estimated our completeness limit for cluster detection to be at $\mathrm{M}_{\mathrm{V}} \sim-6$, so we think we would have picked up a number of the younger objects if they really existed, especially since they would be brighter per unit mass. 